\title{
FMRI ANALYSIS OF COCAINE ADDICTION USING K-SUPPORT SPARSITY
}

\author{
Katerina Gkirtzou ${ }^{1,2}$, Jean Honorio $^{3}$, Dimitris Samaras ${ }^{3}$, Rita Goldstein $^{4}$, Matthew B. Blaschko ${ }^{1,2,5}$ \\ ${ }^{1}$ Center for Visual Computing, École Centrale Paris, France \\ ${ }^{2}$ Équipe Galen, INRIA Saclay, Île-de-France, France \\ ${ }^{3}$ Computer Science Departement, Stony Brook University, Stony Brook, NY, USA \\ ${ }^{4}$ Icahn School of Medicine at Mount Sinai, New York, NY, USA \\ ${ }^{5}$ Université Paris-Est, LIGM (UMR CNRS), École des Ponts ParisTech, France
}

\begin{abstract}
In this paper, we explore various sparse regularization techniques for analyzing fMRI data, such as LASSO, elastic net and the recently introduced $k$-support norm. Employing sparsity regularization allow us to handle the curse of dimensionality, a problem commonly found in fMRI analysis. We test these methods on real data of both healthy subjects as well as cocaine addicted ones and we show that although LASSO has good prediction, it lacks interpretability since the resulting model is too sparse, and results are highly sensitive to the regularization parameter. We find that we can improve prediction performance over the LASSO using elastic net or the $k$-support norm, which is a convex relaxation to sparsity with an $\ell_{2}$ penalty that is tighter than the elastic net. Elastic net and $k$-support norm overcome the problem of overly sparse solutions, resulting in both good prediction and interpretable solutions, while the $k$-support norm gave better prediction performance. Our experimental results support the general applicability of the $k$-support norm in fMRI analysis, both for prediction performance and interpretability.
\end{abstract}

Index Terms - Functional magnetic resonance imaging (fMRI), variable selection, sparsity regularization

\section{INTRODUCTION}

Functional magnetic resonance imaging (fMRI) is a wide spread modality within the field of neuroimaging, that measures brain activity by detecting associated changes in blood flow. The goal of fMRI data analysis is to detect correlations between brain activation and a task the subject performs during the scan. Many statistical methods have been proposed for analyzing fMRI data, including generalized linear model [1, 2], support vector machines [3], independent component analysis $[4,5]$ and kernel canonical correlation analysis $[6,7]$. All these methods have to deal with $(a)$ data that lie

This work is partially funded by the European Research Council under the Seventh Framework Programme (FP7/2007-2013)/ERC Grant 259112. We thank A. Bartels for providing data. J. Honorio is currently with MIT. in a high-dimensional space, with ten of thousands of voxels, (b) a small number of samples, due to the high cost and time consuming nature of the fMRI acquisition procedure, and (c) high levels of noise that arise from different sources, such as system noise and random neural activity.

Sparsity regularizers are key statistical methods for improving predictive performance in the event that the number of observations is substantially smaller than the dimensionality of the data, as is the case in fMRI analysis. The main methods considered here are the LASSO [8], the elastic net [9], and the $k$-support norm [10]. The former two are frequently applied sparsity regularizers developed in the statistics literature, while the latter is a recently introduced method that is mathematically related to the elastic net (cf. Section 2). The former two have previously been applied to fMRI analysis [11], while we are the first to apply the $k$-support norm to the best of our knowledge. We apply these methods to two different real data sets, the first consists of a healthy subject viewing a movie $[4,1,12]$ while the second one consists of both cocaine addicted and healthy non-drug-using subjects performing a monetary reward task [13, 14]. Previous works that have explored sparsity regularization in fMRI are numerous and include $[11,15]$.

The rest of the paper is organized as follows: we first introduce the sparse regularization techniques (Section 2). Then, we present our results on two real data sets (Section 3) and we conclude with a discussion (Section 4).

\section{APPROACH}

\subsection{Sparsity Regularization and the $k$-support Norm}

In the sequel, we assume that we have a sample of labeled training data $\left\{\left(x_{1}, y_{1}\right), \ldots,\left(x_{n}, y_{n}\right)\right\} \in\left(\mathbb{R}^{d} \times \mathbb{R}\right)^{n}$ where $x_{i}$ is the output of a fMRI scan, and $y_{i}$ is a ground truth label that we would like to be able to predict.

A cornerstone of statistical inference is the application of regularized risk, in which a loss function is evaluated over a sample of data and is linearly combined with a regularizer that 
Table 1. A summary of the regularizers considered in this work.

\begin{tabular}{r|c} 
Regularizer & $\Omega(w)$ \\
\hline LASSO [8] & $\lambda_{1}\|w\|_{1}$ \\
Elastic net [9] & $\lambda_{1}\|w\|_{1}+\lambda_{2}\|w\|_{2}^{2}$ \\
$k$-support [10] & $\lambda\|w\|_{k}^{s p}$ (see Equation (2))
\end{tabular}

penalizes some norm of the prediction function. For simplicity, we concern ourselves only with squared error of a scalar output and a linear function class

$$
\arg \min _{w \in \mathbb{R}^{d}} \lambda \Omega(w)+\frac{1}{n} \sum_{i=1}^{n}\left(\left\langle w, x_{i}\right\rangle-y_{i}\right)^{2}
$$

where $\lambda$ is a scalar parameter controling the degree of regularization and $\Omega$ is a scalar valued function monotonic in a norm of $w$. Sparsity regularization is a key family of priors over linear functions that prevents overfitting, and aids interpretability of the resulting models $[8,9,10,11,15]$. Key to the mathematical understanding of sparsity regularizers is their interpretaion as convex relaxations to quantities involving the $\ell_{0}$ norm, which simply counts the number of non-zero elements of a vector. Two of the most important sparsity regularizers, the LASSO [8] and the elastic net [9], are achieved by setting $\Omega$ to be the $\ell_{1}$ norm of $w$ or a linear combination of the $\ell_{1}$ and squared $\ell_{2}$ norms, respectively (Table 1). The elastic net has been employed in situations where there may be multiple correlated signals that should be combined to improve prediction accuracy, a case where the LASSO would yield a higher variance predictor.

The $\ell_{1}$ norm, which is the sum of the absolute values of the vector, has an important interpretation as the convex relaxation of the $\ell_{0}$ norm, meaning it is the tightest sparsity norm that retains convexity, which is key for computational tractability. While the LASSO can therefore be interpreted as employing the convex hull of the $\ell_{0}$ sparsity regularizer, the elastic net is looser than the convex hull of a norm that combines $\ell_{2}$ regularization with sparsity [10]. However, one may employ the $k$-support norm, which is exactly the convex hull of that hybrid norm. The $k$-support norm can be computed as

$$
\|w\|_{k}^{s p}=\left(\sum_{i=1}^{k-r-1}\left(|w|_{i}^{\downarrow}\right)^{2}+\frac{1}{r+1}\left(\sum_{i=k-r}^{d}|w|_{i}^{\downarrow}\right)^{2}\right)^{\frac{1}{2}}
$$

where $|w|_{i}^{\downarrow}$ is the $i$ th largest element of the vector and $r$ is the unique integer in $\{0, \ldots, k-1\}$ satisfying

$$
|w|_{k-r-1}^{\downarrow}>\frac{1}{r+1} \sum_{i=k-r}^{d}|w|_{i}^{\downarrow} \geq|w|_{k-r}^{\downarrow} .
$$

The $k$-support norm is closely related to the elastic net, in that it can be bounded to within a constant factor of the elastic net, but leads to slightly different sparsity patterns. One can see from Equation (2) that the norm trades off a squared $\ell_{2}$ penalty for the largest components with an $\ell_{1}$ penalty for the smallest components. While initial experiments have shown promising results with the $k$-support norm for a range of machine learning problems [10], to the best of our knowledge this study is the first to apply the approach to fMRI.

\section{2. fMRI Analysis of Cocaine Addiction}

The neuropsychological experiment for cocaine addiction data set has a block design, that included six sessions, with each of them having different conditions. The two varying conditions are the monetary reward $(50 \hat{c}, 25 \dot{c}$ and $0 \dot{c})$ and the cue shown (drug words, neutral words). The session consists of an initial screen displaying the monetary reward and then presenting a sequence of forty words in four different colors (yellow, blue, red or green). The subject was instructed to press one of four buttons matching the color of the word they had just read. The subjects were rewarded for correct performance depending on the monetary condition. In this paper, we focus on the monetary conditions only, and more specifically the session of 50c following [14]. The dataset consists of 16 cocaine addicted individuals and 17 control subjects. These were the subjects that complied to the following requirements: motion $<2 \mathrm{~mm}$ translation, $<2^{\circ}$ rotation and at least $50 \%$ performance of the subject in an unrelated task [13]. For each subject a contrast map was computed using the statistical parametric mapping package SPM2 (http://www. fil.ion.ucl.ac.uk/spm/).

\section{RESULTS}

Results are presented on two fMRI datasets. The first consists of a healthy subject in a free-viewing setting. Data collection was previously described in $[4,1]$, while the pre-processing followed [12]. The discriminative task is the prediction of a "Temporal Contrast" variable computed from the content of a movie presented to the subject [7]. This dataset was employed for the quantitative evaluation due to its larger sample size. The second dataset consists of control and cocaine addicted subjects [13, 14], as described in Section 2.

The performance of the different sparse regularization techniques, shown in Figure 1, is evaluated as the mean correlation over 100 trials of random permutation of the data described in [12]. In each trial, $80 \%$ of the data are used to train the method, while the remaining $20 \%$ are used to evaluate the performance. More specifically, Figure 1(a) shows the mean correlation between LASSO and elastic net against the number of non-zero variables (i.e voxels), while Figure 1(b) shows the mean correlation for the $k$-support norm against different $k$ values-which are correlated with the number of non-zero coefficients. LASSO achieves a maximum mean correlation of 0.1198 for 44 non-zero variables, elastic net 


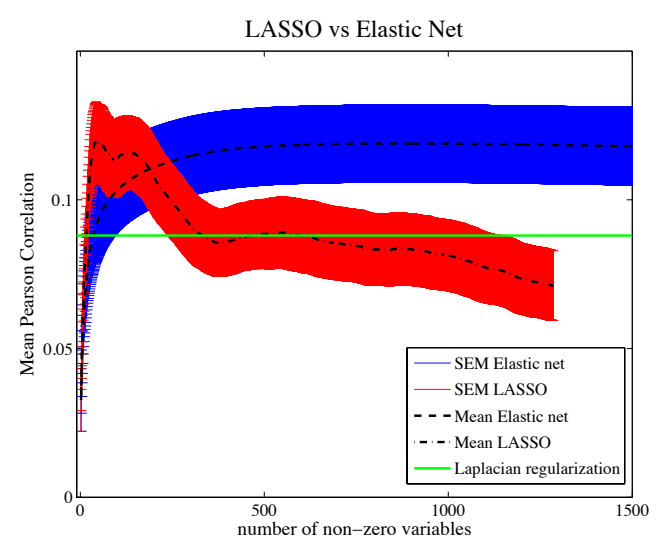

(a) LASSO vs Elastic net

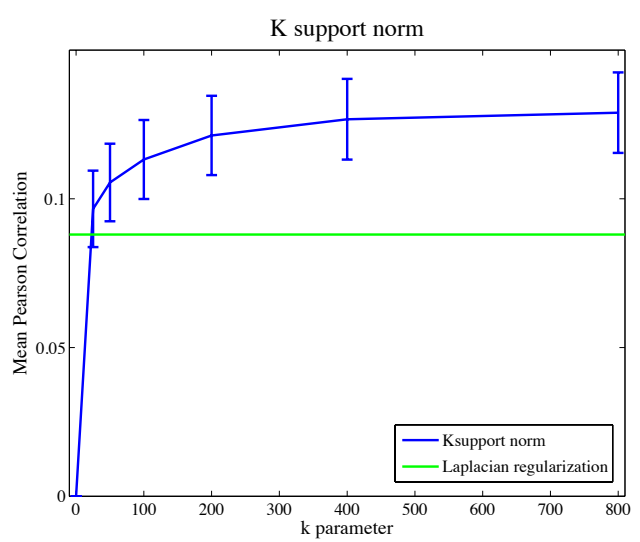

(b) $k$-support norm

Fig. 1. Mean Pearson correlations between the label and prediction on the hold-out data over 100 trials for the dataset described in [12] (higher values indicate better performance). Error bars show the standard deviation. The LASSO achieves its best performance with a sparsity level substantially lower than the elastic net, as it suppresses correlated voxels (Figure 1(a)). The $k$-support norm performs better than the LASSO, elastic net, or Laplacian regularization reported in [7], and is a promising candidate for sparsity in fMRI analysis (Figure 1(b)). (Figure best viewed in color.)

a maximum mean correlation of 0.1189 for 866 non-zero variables, while $k$-support norm a maximum of 0.129 for $k=800$. This is substantially higher than was previously reported in [7].

We have additionally visualized the brain regions predicted when applying the LASSO and the $k$-support norm to the data from $[13,14]$. For each, we have selected slices through the brain that maximize the sum of the absolute values of the weights predicted by the respective methods. These results are presented in Figure 2 and discussed in the next section.

\section{DISCUSSION}

The main area of activity shown in Figure 2(b) is the rostral anterior cingulate cortex (rostral ACC). It has been shown to be deactivated during the drug Stroop as compared to baseline in cocaine users vs. controls even when performance, task interest and engagement are matched between the groups [13] and that its activity is normalized by oral methylphenidate [16]-which similarly to cocaine blocks the dopamine transporters increasing extracellular dopamine-an increase that was associated with lower task-related impulsivity (errors of commission). This region was responsive (showed reduction in drug cue reactivity) to pharmacotherapeutic interventions in cigarette smokers [17, 18], and may be a marker of treatment response in other psychopathology (e.g., depression). The LASSO does not show a meaningful sparsity pattern (Figure 2(a)).

In this work, we have investigated the applicability of sparsity regularizers in fMRI analyses. We have shown that the $k$-support norm can give better predictive performance than the LASSO and elastic net, while having favorable mathematical and computational properties. Furthermore, the brain regions implicated in addiction by the $k$-support norm coincide with previous results on addiction, indicating that the $k$-support norm is additionally useful for generating sparse, but correlated, regions suitable for interpretation in a medical-research setting. In the specific task of cocaine addiction, the $k$-support norm has implicated the involvement of the rostral ACC, in line with previous studies, while the LASSO did not lead to an interpretable result. We therefore consider the $k$-support norm as a promising tool for sparsity in fMRI analysis and believe it merits future study. ${ }^{1}$

\section{REFERENCES}

[1] A. Bartels, S. Zeki, and N.K. Logothetis, "Natural vision reveals regional specialization to local motion and to contrast-invariant, global flow in the human brain," Cereb. Cortex, 2007.

[2] A. Bartels and S. Zeki, "Functional brain mapping during free viewing of natural scenes.", Human Brain Mapping, vol. 21, no. 2, pp. 75-85, 2004.

[3] S. Song, Z. Zhan, Z. Long, J. Zhang, and L. Yao, "Comparative study of svm methods combined with voxel selection for object category classification on fmri data.," PLoS One, vol. 6, no. 2, pp. e17191, 2011.

[4] A. Bartels and S. Zeki, "The chronoarchitecture of the human brain-natural viewing conditions reveal a time-

\footnotetext{
${ }^{1}$ Code for the $k$-support norm is available at http://www . centrale-ponts.fr/personnel/gkirtzou/code/.
} 


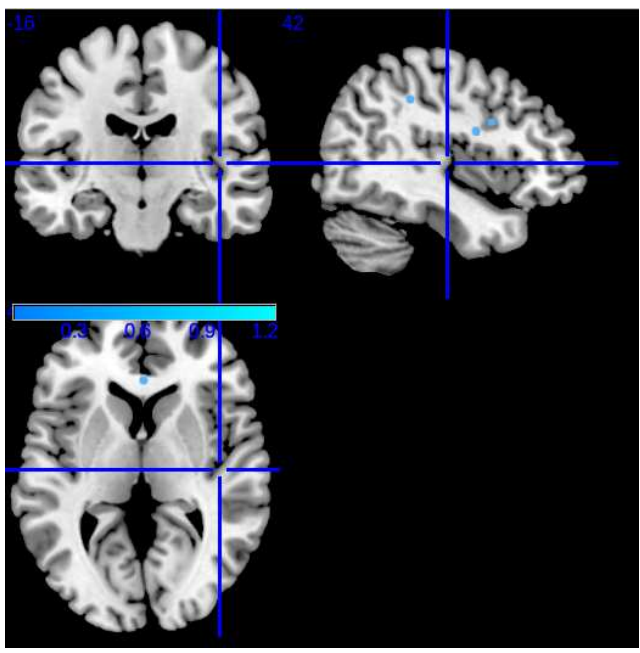

(a) LASSO

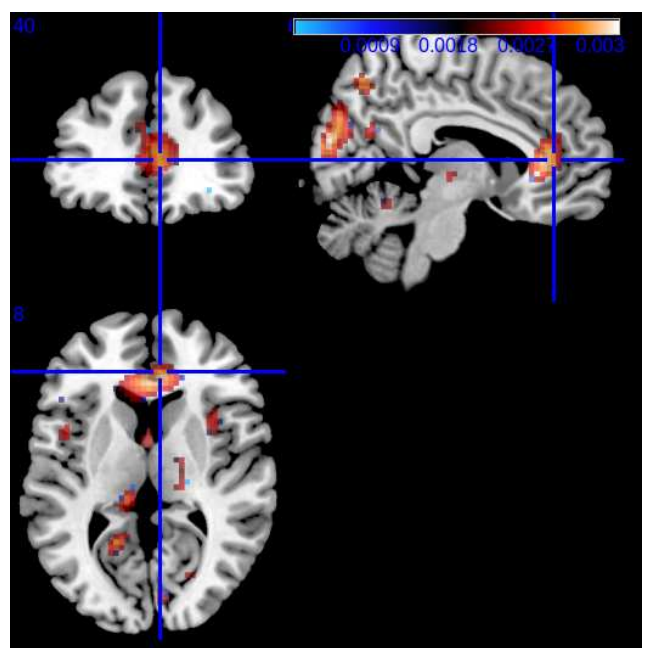

(b) $k$-support norm

Fig. 2. A visualization of the areas of the brain selected by the LASSO and by the $k$-support norm applied to the data described in [13]. The LASSO leads to overly sparse solutions that do not lend themselves to easy interpretation (Figure 2(a)), while the $k$-support norm does not suppress correlated voxels, leading to interpretable and robust solutions (Figure 2(b)). A medical interpretation of the result presented in Figure 2(b) is given in Section 4. (Figure best viewed in color.)

based anatomy of the brain," NeuroImage, vol. 22, no. 1, pp. $419-433,2004$.

[5] A. Bartels and S. Zeki, "Brain dynamics during natural viewing conditions-a new guide for mapping connectivity in vivo," Neurolmage, vol. 24, no. 2, pp. 339-349, 2005.

[6] D.R. Hardoon, J. Mourão-Miranda, M. Brammer, and J. Shawe-Taylor, "Unsupervised analysis of fMRI data using kernel canonical correlation," NeuroImage, vol. 37, no. 4, pp. 1250 - 1259, 2007.

[7] M.B. Blaschko, J.A. Shelton, A. Bartels, C.H. Lampert, and A. Gretton, "Semi-supervised kernel canonical correlation analysis with application to human fMRI," Pattern Recognition Letters, vol. 32, no. 11, pp. 1572 1583, 2011.

[8] R. Tibshirani, "Regression shrinkage and selection via the lasso," Journal of the Royal Statistical Society Series B, vol. 58, pp. 267-288, 1996.

[9] H. Zou and T. Hastie, "Regularization and variable selection via the elastic net," Journal of the Royal Statistical Society Series B, vol. 67, no. 2, pp. 301-320, 2005.

[10] A. Argyriou, R. Foygel, and N. Srebro, "Sparse prediction with the k-support norm," in NIPS. 2012.

[11] M.K. Carroll, G.A. Cecchi, I. Rish, R. Garg, and A.R. Rao, "Prediction and interpretation of distributed neural activity with sparse models," NeuroImage, vol. 44, no. 1, pp. $112-122,2009$.
[12] M.B. Blaschko, J.A. Shelton, and A. Bartels, "Augmenting feature-driven fMRI analyses: Semi-supervised learning and resting state activity," in NIPS. 2009.

[13] R.Z. Goldstein, N. Alia-Klein, D. Tomasi, J.H. Carrillo, T. Maloney, P.A. Woicik, R. Wang, F. Telang, and N.D. Volkow, "Anterior cingulate cortex hypoactivations to an emotionally salient task in cocaine addiction," PNAS, vol. 106, no. 23, pp. 9453, 2009.

[14] J. Honorio, D. Tomasi, R. Goldstein, H.C. Leung, and D. Samaras, "Can a single brain region predict a disorder?," IEEE Transactions on Medical Imaging, 2012.

[15] B. Ng, V. Siless, G. Varoquaux, J.-B. Poline, B. Thirion, and R. Abugharbieh, "Connectivity-informed sparse classifiers for fMRI brain decoding," in Pattern Recognition in Neuroimaging, 2012.

[16] R.Z. Goldstein, P.A. Woicik, T. Maloney, et al., "Oral methylphenidate normalizes cingulate activity in cocaine addiction during a salient cognitive task," PNAS, vol. 107, no. 38, pp. 16667-16672, 2010.

[17] Culbertson CS, Bramen J, Cohen MS, et al., "Effect of bupropion treatment on brain activation induced by cigarette-related cues in smokers," Archives of General Psychiatry, vol. 68, no. 5, pp. 505-515, 2011.

[18] T.R. Franklin, Z. Wang, Y. Li, et al., "Dopamine transporter genotype modulation of neural responses to smoking cues: confirmation in a new cohort," Addiction Biology, vol. 16, no. 2, pp. 308-322, 2011. 